The Free Internet Journal

for Organic Chemistry
Paper

Arkivoc 2017, part iii, 287-292

\title{
Tert-butylation of naphthalene-2,6-diol and 6-methoxynaphthalen-2-ol
}

\author{
Andrey A. Berezin* and Davide Marinelli \\ School of Chemistry, Cardiff University Main Building, Park Pl, Cardiff CF10 3AT, United Kingdom \\ Email: Berezina@cardiff.ac.uk
}

Dedicated to Professor (Dr.) Oleg A. Rakitin on the occasion of his $65^{\text {th }}$ birthday

Received 06-30-2017

Accepted 07-19-2017

Published on line $08-23-2017$

\section{Abstract}

The products of $t$-butylation of naphthalene-2,6-diol and 6-methoxynaphthalen-2-ol with isobutylene and $t$ butanol were identified and characterized; the structures of those products were proved by single crystal Xray structural analysis.)

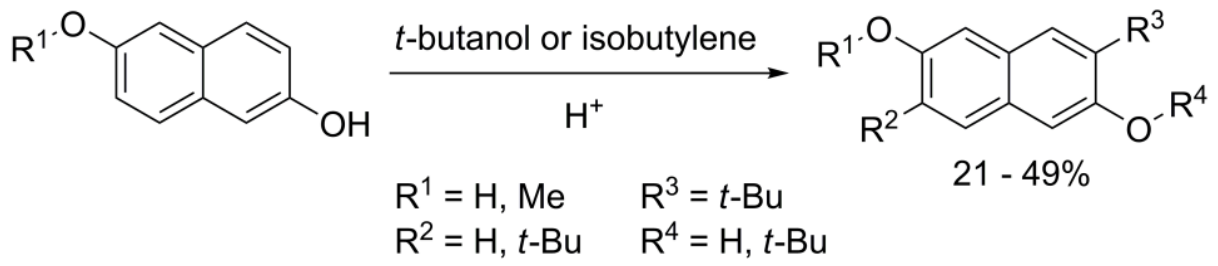

Keywords: X-ray, butylation, naphthol, napththalenediol, methoxynaphthol 


\section{Introduction}

The $t$-butylation of $\alpha$ - and $\beta$-naphthols has been studied since the middle of $20^{\text {th }}$ century. There are three general methods for $t$-butylation reported in the literature: Friedel-Crafts alkylation with $t$-butyl halide in the presence of Lewis acid, alkylation with isobutylene or with $t$-butanol in the presence of protic acids. The assignment of the position of $t$-butyl groups presented major difficulties during past studies and led to many mistakes and non-reproducible results. ${ }^{1,2}$ Nowadays the chemistry of $t$-butylation of $\alpha$-and $\beta$-naphthols is well established and the structures of the products have been proved by 2D NMR. ${ }^{3,4}$ Only the $t$-butylation of $1,5-$ naphthalenediol ${ }^{5}$ and 2,3-naphthalenediol ${ }^{6,7}$ have been reported in literature to give 2,6-di-tertbutylnaphthalene-1,5-diol and 6-tert-butylnaphthalene-2,3-diol respectively (Scheme 1).
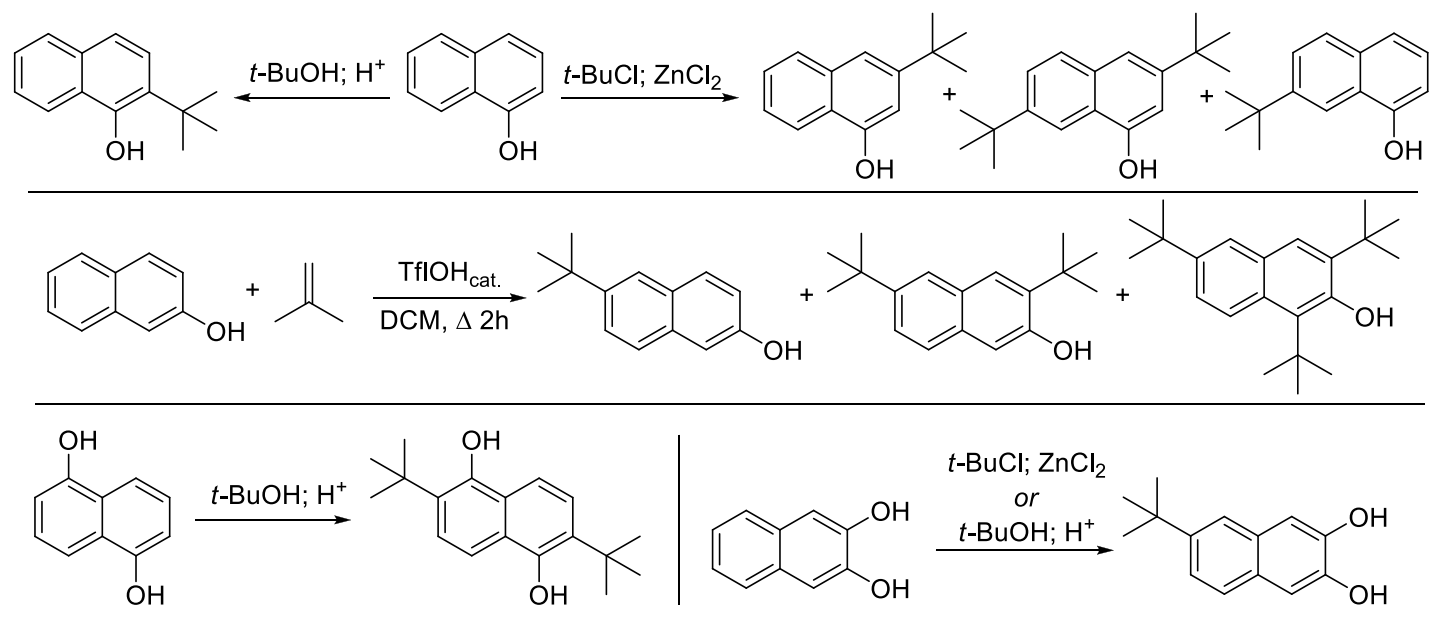

Scheme 1. Published syntheses of tert-butyl naphthols.

\section{Results and Discussion}

We recently required 3,7-di-tert-butyl-6-methoxynaphthalen-2-ol as a building block for synthesis of soluble derivatives of peri-xanthenoxanthenes. We decided to use naphthalene-2,6-diol as the starting material and to try the conditions reported for $t$-butylation of 2-naphthol that involves isobutylene in dichloromethane in the presence of catalytic amount of triflic acid. ${ }^{3}$ The desired 3,7-di-tert-butyInaphthalene-2,6-diol (2) was obtained in this reaction with only $5 \%$ yield mainly due to poor solubility of naphthalene-2,6-diol (1) in dichloromethane. Starting naphthalene-2,6-diol (1) was recovered in $78 \%$ yield together with quinoidal products of oxidation besides the desired product $\mathbf{2}$ that has been isolated, and its structure confirmed by single crystal X-ray structural analysis (Scheme 2 ).

3,7-Di-tert-butylnaphthalene-2,6-diol (2) proved to be stable in TFA solution up to $40^{\circ} \mathrm{C}$ and up to $70{ }^{\circ} \mathrm{C}$ in TFA in the presence of excess of $t$-butanol. At higher temperatures, it starts to lose $t$-butyl groups returning to naphthalene-2,6-diol (1). 3,7-Di-tert-butylnaphthalene-2,6-diol (2) appeared to be unstable in solution to oxidation by air and quickly gave deep blue colored products upon day light irradiation.

Naphthalene-2,6-diol (1) produced 3,7-di-tert-butylnaphthalene-2,6-diol (2) in 12\% yield together with unreacted naphthalene-2,6-diol (1) and products of its oxidation on heating at $60^{\circ} \mathrm{C}$ with excess of $t$-butanol and TFA for 4 hours. Attempt to substitute $t$-butanol by isobutylene in this reaction did not give any products and only returned unreacted naphthalene-2,6-diol (1). Nevertheless, the addition of catalytic amount of triflic 
acid into the reaction mixture, preheated to $70{ }^{\circ} \mathrm{C}$ and left to cool down slowly to ambient temperature upon addition of isobutylene afforded 3,7-di-tert-butylnaphthalene-2,6-diol (2) in 21\% yield together with unreacted naphthalene-2,6-diol (1) and 3-tert-butylnaphthalene-2,6-diol (3) in 20\% yield. The longer reaction time does only increase the level of products of oxidation and decrease the yields of naphthols (Scheme 3).
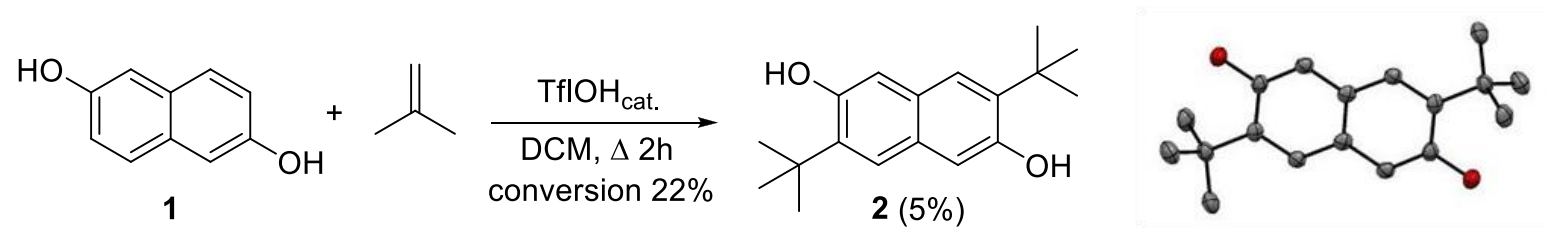

Scheme 2. Left: reaction of naphthalene-2,6-diol (1) with isobutylene. Right: ORTEP representation of 3,7-ditert-butylnaphthalene-2,6-diol (2) determined by X-ray diffraction (atom colors: $\mathrm{C}$ in black, $\mathrm{O}$ in red. Hydrogen atoms omitted for clarity). Space group: I 2/a. Atomic displacement parameters, obtained at $150 \mathrm{~K}$, are drawn at the $50 \%$ probability level.

a).<smiles>Oc1ccc2cc(O)ccc2c1</smiles>

1

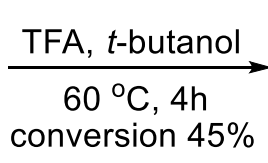
$60^{\circ} \mathrm{C}, 4 \mathrm{~h}$
conversion $45 \%$

$+\underset{10{ }^{\circ} \mathrm{C} \text { to r.t., } 1 \mathrm{~h}}{\stackrel{\mathrm{TFA}, \mathrm{TflOH}_{\text {cat. }}}{\longrightarrow}}$

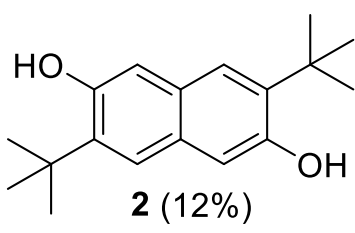

$2(12 \%)$ c).

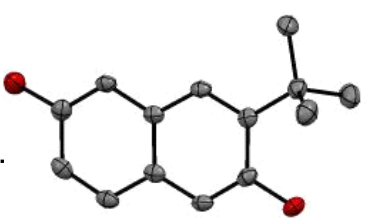

b).

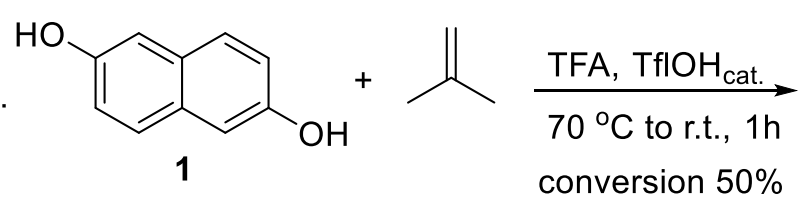<smiles>CC(C)(C)c1cc2cc(O)c(C(C)(C)C)cc2cc1O</smiles>

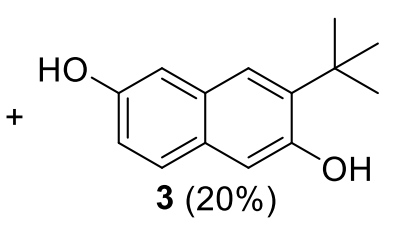

Scheme 3. Reaction of naphthalene-2,6-diol (1) with $t$-butanol (a) and isobutylene (b) in TFA. (c). ORTEP representation of 3-tert-butylnaphthalene-2,6-diol (3) determined by X-ray diffraction (atom colors: $\mathrm{C}$ in black, $\mathrm{O}$ in red. Hydrogen atoms omitted for clarity). Space group: P 2 1 /c. Atomic displacement parameters, obtained at $293 \mathrm{~K}$, are drawn at the $50 \%$ probability level.

In order to overcome the problem of solubility of starting material we selected much more soluble 6methoxynaphthalen-2-ol (4) in reaction with $t$-butanol in dichloromethane/TFA solution at $40^{\circ} \mathrm{C}$ that delivered 3,7-di-tert-butyl-6-methoxynaphthalen-2-ol (5) in 49\% yield together with unreacted starting naphthol 4, 3tert-butyl-6-methoxynaphthalen-2-ol (6) and the product of demethylation - 3,7-di-tert-butylnaphthalene-2,6diol (2) as a minor product (Scheme 4).

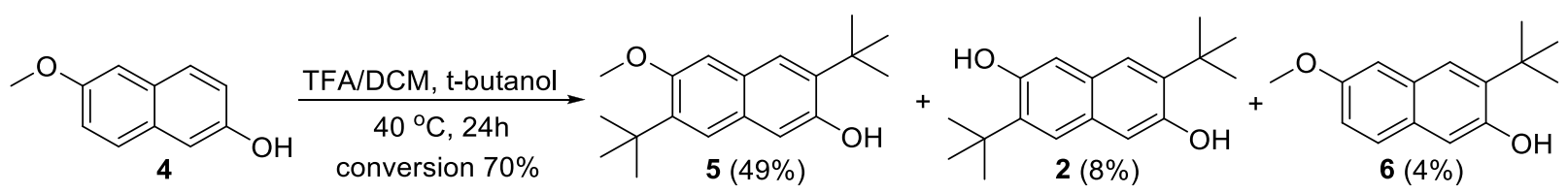

Scheme 4. Reaction of 6-methoxynaphthalen-2-ol (4) with t-butanol in DCM/TFA. The structure of 3,7-di-tertbutyl-6-methoxynaphthalen-2-ol (5) was confirmed by single crystal X-ray diffraction (Figure 1). 


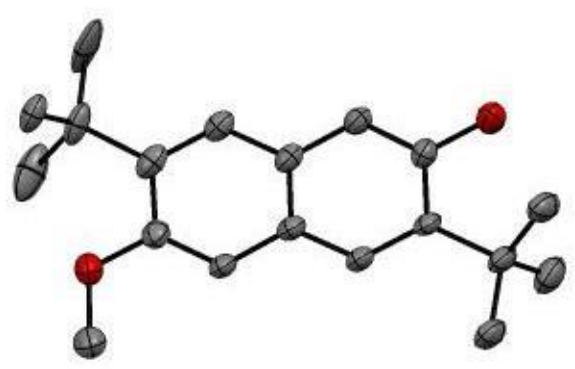

Figure 1. ORTEP representation of 3,7-di-tert-butyl-6-methoxynaphthalen-2-ol (5) determined by X-ray diffraction (atom colors: $\mathrm{C}$ in black, $\mathrm{O}$ in red. Hydrogen atoms omitted for clarity). Space group: $\mathrm{P}$ b c $\mathrm{n}$. Atomic displacement parameters, obtained at $150 \mathrm{~K}$, are drawn at the $50 \%$ probability level.

The attempt to obtain 3,7-di-tert-butyl-6-methoxynaphthalen-2-ol (5) from 6-methoxynaphthalen-2-ol (4) with isobutylene in dichloromethane and catalytic amount of triflic acid unexpectedly gave 2-tert-butoxy-6methoxynaphthalene (7) in reasonably good yield (based on reacted starting material) (Scheme 5).

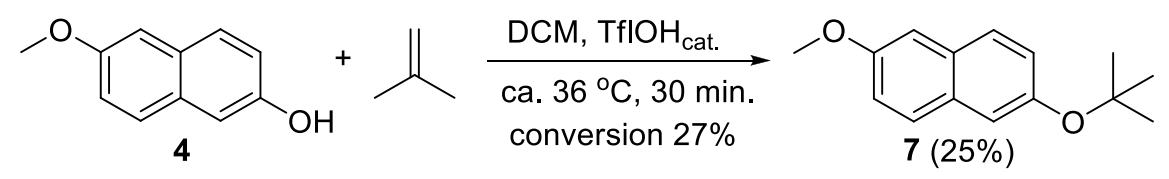

Scheme 5. Reaction of 6-methoxynaphthalen-2-ol (4) with isobutylene in DCM with catalytic amount of triflic acid.

2,6-Dimethoxynaphthalene was tested with isobutylene and $t$-butanol under conditions listed above but did not give products of $t$-butylation.

\section{Conclusions}

$t$-Butylation of naphthalene-2,6-diol and 6-methoxynaphthalen-2-ol has been successfully developed and the structure of products obtained unambiguously determined by single crystal X-ray structural analysis.

\section{Experimental Section}

General.Thin layer chromatography (TLC) was conducted on pre-coated aluminum sheets with $0.20 \mathrm{~mm}$ Machevery-Nagel Alugram SIL G/UV254 with fluorescent indicator UV254. Column chromatography was carried out using Merck Gerduran silica gel 60 (particle size 63-200 $\mu \mathrm{m}$ ). Nuclear magnetic resonance (NMR) ${ }^{1} \mathrm{H}$, and ${ }^{13} \mathrm{C}$ spectra were obtained on a $300 \mathrm{MHz}$ (Bruker) and $400 \mathrm{MHz}$ (Bruker AVANCE III HD) NMR at rt. Chemical shifts were reported in ppm according to tetramethylsilane using the solvent residual signal as an internal reference $\left(\mathrm{CDCl}_{3}: \delta_{\mathrm{H}} 7.26 \mathrm{ppm}, \delta_{\mathrm{C}} 77.16 \mathrm{ppm}\right.$; acetone- $\left.d_{6}: \delta_{\mathrm{H}} 2.05 \mathrm{ppm}, \delta_{\mathrm{C}} 29.84 \mathrm{ppm}\right)$. Coupling constants $(J)$ were given in $\mathrm{Hz}$. Resonance multiplicity was described as $s$ (singlet), $d$ (doublet), $d d$ (doublet of doublets) and $b r$ (broad signal). Carbon spectra were acquired with a complete decoupling for the proton. The 
Attached Proton Test (APT) experiments were used to determine $\mathrm{C}-\mathrm{H}$ multiplicities in carbon spectras. Infrared spectra (IR) were recorded on a Shimadzu IR Affinity 1S FTIR spectrometer in ATR (attenuated total reflection) mode with a diamond mono-crystal. Mass spectrometry was performed by the high-resolution electron ionisation mass spectra (HRMS) on a Waters GCT Premier mass spectrometer with a direct insertion probe in the positive ion mode. The data was processed using the Waters MassLynx software. The commercially available starting materials were used as received without further purification. Isobutylene was obtained according to the literature procedure. ${ }^{8}$

3,7-Di-tert-butylnaphthalene-2,6-diol 2 and 3-tert-butylnaphthalene-2,6-diol (3). Naphthalene-2,6-diol (1) $(1.00 \mathrm{~g}, 6.24 \mathrm{mmol})$ was dissolved in TFA $(100 \mathrm{~mL})$ at $c a .70{ }^{\circ} \mathrm{C}$ and triflic acid $(0.03 \mathrm{~mL}, 0.31 \mathrm{mmol})$ was added. Isobutylene was bubbled through the reaction mixture and the temperature of the reaction mixture slowly decreased to $\mathrm{ca} .40^{\circ} \mathrm{C}$ over 1 hour. The volatiles were removed under reduced pressure at $40{ }^{\circ} \mathrm{C}$, the solid residue dispersed in DCM and the unreacted starting naphthalene-2,6-diol (1) (0.5 g, 50\%) removed by filtration. The filtrate was chromatographed on silica (DCM) to give firstly 3,7-di-tert-butylnaphthalene-2,6diol (2) as colorless solid (0.37 g, 21\%), mp 227-229 ${ }^{\circ} \mathrm{C}$ (from $\mathrm{CHCl}_{3}$ ); $R_{\mathrm{f}}(\mathrm{DCM} /$ petroleum ether 1:1) 0.16; $\mathrm{v}_{\max } / \mathrm{cm}^{-1} 3537 \mathrm{~m}(\mathrm{OH}), 3354 \mathrm{~m}$ (Ar), 2999w (Alk), 2957m (Alk), 2916w (Alk), 2870w (Alk); $\delta_{\mathrm{H}}\left(300 \mathrm{MHz} \mathrm{CDCl}_{3}\right)$ $7.46(2 \mathrm{H}, \mathrm{s}, \mathrm{ArH}), 6.93(2 \mathrm{H}, \mathrm{s}, \mathrm{ArH}), 4.86(2 \mathrm{H}, \mathrm{br} . \mathrm{s}, \mathrm{OH}), 1.47(18 \mathrm{H}, \mathrm{s}, 2 \mathrm{t}-\mathrm{Bu}) ; \delta_{\mathrm{c}}\left(75 \mathrm{MHz}\right.$, acetone-d $\left.d_{6}\right)$ 153.4, 138.8, 129.0, 123.4, 111.1, 35.5, 30.1; HRMS (TOF El+): $\mathrm{m} / \mathrm{z}[\mathrm{M}]+$ calcd for $\left(\mathrm{C}_{18} \mathrm{H}_{24} \mathrm{O}_{2}\right)$ : 272.1776; found: 272.1785. Further elution (DCM) gave 3-tert-butylnaphthalene-2,6-diol (3) $(0.27 \mathrm{~g}, 20 \%)$ as colorless solid, $\mathrm{mp}$ $160-162{ }^{\circ} \mathrm{C}$ (from DCM); $R_{\mathrm{f}}(\mathrm{DCM}) 0.16 ; \mathrm{v}_{\mathrm{max}} / \mathrm{cm}^{-1} 3549 \mathrm{~m}(\mathrm{OH}), 3532 \mathrm{~s}(\mathrm{OH}), 2963 \mathrm{~m}$ (Alk), 2953m (Alk), $2914 \mathrm{~m}$ (Alk), 2876w (Alk); $\delta_{H}\left(400 \mathrm{MHz}, \mathrm{CDCl}_{3}\right) 7.54(1 \mathrm{H}, \mathrm{s}, \mathrm{ArH}), 7.50(1 \mathrm{H}, \mathrm{d}, J$ 8.7, $\mathrm{ArH}), 7.08(1 \mathrm{H}, \mathrm{d}, J 2.4, \operatorname{ArH}), 7.01$ $\left(1 \mathrm{H}, \mathrm{dd}, J\right.$ 8.7, 2.5, ArH), $4.93(2 \mathrm{H}, \mathrm{br} . \mathrm{s}, \mathrm{OH}), 1.49(9 \mathrm{H}, \mathrm{s}, t-\mathrm{Bu}) ; \delta_{\mathrm{c}}(\mathrm{APT}, 75 \mathrm{MHz}$, acetone-d6) 153.8 (s), $153.4(\mathrm{~s})$, 139.5 (s), 130.6 (s), 128.9 (s), 127.2 (d), 124.5 (d), 118.6 (d), 110.9 (d), 110.0 (d), 35.6 (Cq t-Bu), 30.0 (t-Bu); HRMS (TOF El+): $m / z$ [M]+ calcd for $\left(\mathrm{C}_{14} \mathrm{H}_{16} \mathrm{O}_{2}\right): 216.1150$; found: 216.1153 .

3,7-Di-tert-butyl-6-methoxynaphthalen-2-ol (5) and 3-tert-butyl-6-methoxynaphthalen-2-ol (6). 6Methoxynaphthalen-2-ol (4) (1.00 g, $5.74 \mathrm{mmol})$ was dissolved in TFA (10 mL), DCM (5 mL) and tert-butanol $(5.5 \mathrm{~mL})$. The reaction mixture was heated at $c a .40^{\circ} \mathrm{C}$ for 24 hours, the volatiles were removed under reduced pressure at $40{ }^{\circ} \mathrm{C}$, the solid residue dissolved in DCM and chromatographed on silica (DCM) to give firstly 3,7di-tert-butyl-6-methoxynaphthalen-2-ol (5) as colorless solid (0.81 g, 49\%), mp 129-133 ${ }^{\circ} \mathrm{C}$ (from petroleum ether); $R_{\mathrm{f}}(\mathrm{DCM} /$ petroleum ether $1: 1) 0.31 ; \mathrm{v}_{\mathrm{max}} / \mathrm{cm}^{-1} 3499 \mathrm{~m}(\mathrm{OH}), 3003 \mathrm{w}$ (Alk), 2959m (Alk), 2941m (Alk), 2909m (Alk), 2866w (Alk); $\delta_{\mathrm{H}}\left(300 \mathrm{MHz}, \mathrm{CDCl}_{3}\right) 7.58(1 \mathrm{H}, \mathrm{s}, \mathrm{ArH}), 7.46(1 \mathrm{H}, \mathrm{s}, \mathrm{ArH}), 7.09(1 \mathrm{H}, \mathrm{s}, \mathrm{ArH}), 6.93(1 \mathrm{H}, \mathrm{s}$, $\operatorname{ArH}), 4.87(1 \mathrm{H}$, br.s, $\mathrm{OH}), 3.92\left(3 \mathrm{H}, \mathrm{s}, \mathrm{CH}_{3}\right), 1.50(9 \mathrm{H}, \mathrm{s}, t-\mathrm{Bu}), 1.45(9 \mathrm{H}, \mathrm{s}, t-\mathrm{Bu}) ; \delta_{\mathrm{C}}\left(\mathrm{APT}, 75 \mathrm{MHz}, \mathrm{CDCl}_{3}\right) 156.1$ (s), 151.6 (s), 139.8 (s), 137.9 (s), 128.2 (s), 127.8 (s), 123.9 (d), 122.7 (d), 110.8 (d), 106.3 (d), $54.9\left(\mathrm{CH}_{3}\right), 35.2$ (Cq t-Bu), 35.0 (Cq t-Bu), 29.9 (t-Bu), 29.8 (t-Bu); HRMS (TOF El+): $m / z[M]+$ calcd for $\left(C_{19} H_{26} \mathrm{O}_{2}\right): 286.1933$; found: 286.1930 . Further elution (DCM) gave 3,7-di-tert-butylnaphthalene-2,6-diol (2) as colorless solid (0.12 $\mathrm{g}, 8 \%$ ), $\mathrm{mp} 227-229{ }^{\circ} \mathrm{C}$ (from $\mathrm{CHCl}_{3}$ ); $R_{\mathrm{f}}(\mathrm{DCM} /$ petroleum ether $1: 1) 0.16 ; \mathrm{v}_{\mathrm{max}} / \mathrm{cm}^{-1} 3537 \mathrm{~m}(\mathrm{OH}), 3354 \mathrm{~m}(\mathrm{Ar})$, 2999w (Alk), 2957m (Alk), 2916w (Alk), 2870w (Alk); $\delta_{\mathrm{H}}\left(300 \mathrm{MHz}, \mathrm{CDCl}_{3}\right) 7.46$ (2H, s, ArH), $6.93(2 \mathrm{H}, \mathrm{s}, \mathrm{ArH})$, $4.86(2 \mathrm{H}$, br.s, $\mathrm{OH}), 1.47(18 \mathrm{H}, \mathrm{s}, 2 \mathrm{t}$-Bu); identical to the product described above. Further elution (DCM) gave 3-tert-butyl-6-methoxynaphthalen-2-ol (6) as colorless solid (0.05 g, 4\%), mp 65-66 ${ }^{\circ} \mathrm{C} ; R_{\mathrm{f}}(\mathrm{DCM}) 0.19 ; \mathrm{v}_{\max } / \mathrm{cm}^{-}$ ${ }^{1}$ 3254w (Ar), 2999w (Alk), 2957m (Alk), 2916m (Alk), 2868w (Alk), 2833w (Alk); $\delta_{\mathrm{H}}\left(300 \mathrm{MHz}, \mathrm{CDCl}_{3}\right) 7.60(1 \mathrm{H}$, d, J 8.8, $\operatorname{ArH}), 7.52(1 \mathrm{H}, \mathrm{s}, \operatorname{ArH}), 7.09-7.06(2 \mathrm{H}, \mathrm{m}, \mathrm{ArH}), 7.02(1 \mathrm{H}, \mathrm{dd}, J$ 8.8, 2.6, $\operatorname{ArH}), 4.92(1 \mathrm{H}, \mathrm{br} . \mathrm{s}, \mathrm{OH}), 3.92$ $\left(3 \mathrm{H}, \mathrm{s}, \mathrm{CH}_{3}\right), 1.45(9 \mathrm{H}, \mathrm{s}, t-\mathrm{Bu}) ; \delta_{\mathrm{C}}\left(\mathrm{APT}, 75 \mathrm{MHz}, \mathrm{CDCl}_{3}\right) 156.1$ (s), 151.6 (s), 140.5 (s), 129.4 (s), 128.2 (s), 127.5 (d), 123.9 (d), 117.2 (d), 109.7 (d), 106.1 (d), $54.9\left(\mathrm{CH}_{3}\right), 35.2$ (Cq t-Bu), 29.8 (t-Bu); HRMS (TOF El+): $m / z$ [M]+ 
calcd for $\left(\mathrm{C}_{15} \mathrm{H}_{18} \mathrm{O}_{2}\right)$ : 230.1307; found: 230.1308. Further elution (DCM) gave unreacted 6-Methoxynaphthalen2-ol (4) (0.30 g, 30\%).

2-tert-Butoxy-6-methoxynaphthalene (7). 6-Methoxynaphthalen-2-ol (4) (1.00 g, $5.74 \mathrm{mmol}$ ) was dissolved in $\operatorname{DCM}(25 \mathrm{~mL})$ and a triflic acid $(0.05 \mathrm{~mL}, 0.57 \mathrm{mmol})$ was added. Isobutylene was bubbled through the boiling reaction mixture for 30 minutes. The volatiles were removed under reduced pressure at $40{ }^{\circ} \mathrm{C}$ and the solid residue was extracted with hot petroleum ether $(50 \mathrm{~mL})$. The solid remained was unreacted 6methoxynaphthalen-2-ol (4) (0.73 g, 73\%). The petroleum ether extract was chromatographed on silica (DCM) to give 2-tert-butoxy-6-methoxynaphthalene (7) as colorless solid (0.33 g, 25\%), mp 69-70 ${ }^{\circ} \mathrm{C} ; R_{\mathrm{f}}(\mathrm{DCM}) 0.52$; $v_{\max } / \mathrm{cm}^{-1} 2974 \mathrm{~m}$ (Alk), 2932w (Alk), 2903w (Alk); $\delta_{\mathrm{H}}\left(300 \mathrm{MHz}, \mathrm{CDCl}_{3}\right)$ 7.67-7.60 (2H, m, $\left.\operatorname{ArH}\right), 7.34$ (1H, d, J 2.1, $\operatorname{ArH})$, 7.18-7.09 (3H, m, ArH), $3.89\left(3 \mathrm{H}, \mathrm{s}, \mathrm{CH}_{3}\right), 1.38(9 \mathrm{H}, \mathrm{s}, t-\mathrm{Bu}) ; \delta_{\mathrm{C}}\left(\mathrm{APT}, 75 \mathrm{MHz}, \mathrm{CDCl}_{3}\right) 156.7$ (s), 151.2 (s), 131.3 (s), 129.3 (s), 128.6 (d), 127.2 (d), 125.5 (d), 120.3 (d), 118.8 (d), 105.5 (d), 78.6 (Cq t-Bu), $55.3\left(\mathrm{CH}_{3}\right)$, $28.8(t-\mathrm{Bu})$; HRMS (TOF El+): $m / z$ [M]+ calcd for $\left(\mathrm{C}_{15} \mathrm{H}_{18} \mathrm{O}_{2}\right): 230.1307$; found: 230.1313 .

\section{Acknowledgements}

We are grateful to ERC Starting Grant “COLORLANDS” project for financial support and to Prof. Davide Bonifazi for providing us a laboratory, equipment, consumables and scientific expertise.

\section{Supplementary Material}

${ }^{1} \mathrm{H},{ }^{13} \mathrm{C}$ NMR spectra and crystallographic data of reported compounds can be found in supplementary materials.

\section{References}

1. Buu-Hol̈, N. P.; Bihan, H. L.; Binon, F.; Rayet, P. J. Org. Chem. 1950, 15, $1060-1066$. http://dx.doi.org/10.1021/jo01151a024

2. Miyata, T. ; Hamada, T. ; Hirashima, T. ; Manabe, O. ; Hiyama, H. J. Syn. Org. Chem., Japan 1973, 31, 831-834. http://dx.doi.org/10.5059/yukigoseikyokaishi.31.831

3. Braddock, C. D.; Tucker, S. C.; Brown, J. M. Bull.Soc. Chim. Fr. 1997, 399-410.

4. Georghiou, P. E.; Ashram, M. J. Org. Chem. 1995, 60, 2909-2911. http://dx.doi.org/10.1021/jo00114a049

5. Christensen, J. B.; Bechgaard, K. J. Heterocycl. Chem. 2003, 40, 757-761. http://dx.doi.org/10.1002/jhet.5570400503

6. Marinina, L. E.; Alekseeva, V. I.; Sawina, L. P.; Luk'yanets, E. A. Chem. Heterocycl. Compounds 1988, 24, $220-225$. http://dx.doi.org/10.1007/BF00473337

7. Taylor, R. G. D.; Bezzu, C. G.; Carta, M.; Msayib, K. J.; Walker, J.; Short, R.; Kariuki, B. M.; McKeown, N. B. Chemistry - A European Journal 2016, 22, 2466-2472.

http://dx.doi.org/10.1002/chem.201504212

8. Vanino, L. Handbuch der präparativen Chemie; ein Hilfsbuch für das arbeiten im chemischen Laboratorium unter Mitwirkung verschiedener Fachgenossen, Vol. 2, Ferdinand Enke, Stuttgart, 1923.

http://books.google.com/books?id=zvg4AAAAIAAJ\&ie=ISO-8859-1 